\title{
Energy Relaxation and Structural Dynamics of Protons in Water/ DMSO Mixtures
}

\author{
Oleksandr O. Sofronov*(i) and Huib J. Bakker \\ AMOLF, Science Park 104, 1098 XG Amsterdam, The Netherlands \\ Supporting Information
}

ABSTRACT: We investigate the structure and dynamics of proton solvation structures in mixed water/dimethyl sulfoxide (DMSO) solvents using two-color mid-infrared femtosecond pump-probe spectroscopy. At a water fraction below 20\%, protons are mainly solvated as $(\mathrm{DMSO}-\mathrm{H})^{+}$and (DMSO$\mathrm{H})^{+}-\mathrm{H}_{2} \mathrm{O}$ structures. We find that excitation of the $\mathrm{OH}$-stretch vibration of the proton in (DMSO-H $)^{+}-\mathrm{H}_{2} \mathrm{O}$ structures leads to an ultrafast contraction of the hydrogen bond between (DMSO-H) ${ }^{+}$and $\mathrm{H}_{2} \mathrm{O}$. This excited state relaxes rapidly with $T_{1}=95 \pm 10 \mathrm{fs}$ and leads in part to a strong local heating effect and in part to predissociation of the protonated cluster into $(\mathrm{DMSO}-\mathrm{H})^{+}$and water monomers.

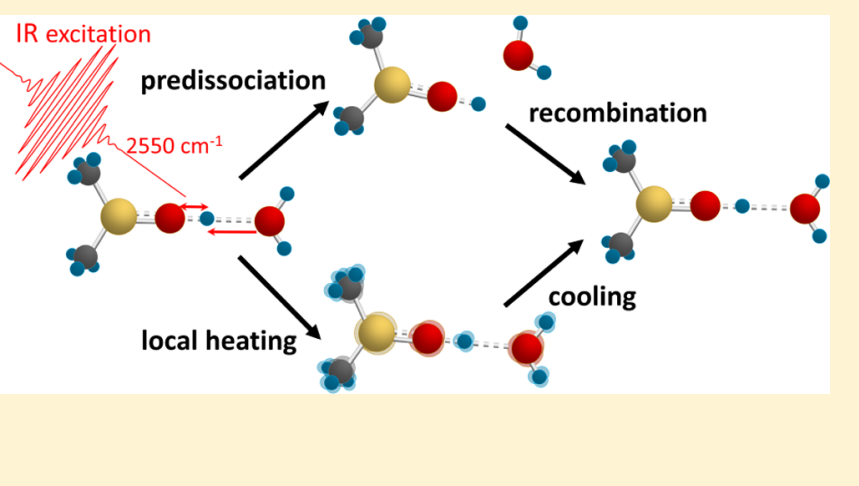

in turn report on changes in the covalent and hydrogen bonds. ${ }^{16}$ In recent years, several femtosecond mid-infrared studies on the hydrated proton have been reported. ${ }^{9,17-19}$ In these experiments, the dynamics of proton solvation in different aqueous media (water, water/acetonitrile, and hydrated Nafion membranes) was studied by means of excitation of the hydrated proton $\mathrm{OH}$-stretch vibrations. These studies showed that ultrafast structural fluctuations of the hydrated proton result in a sub- 100 fs vibrational relaxation of the vibrationally excited state. Only for the $\mathrm{H}_{3} \mathrm{O}^{+}$-ion strongly bound to a negatively charged sulfonate in Nafion membranes, the vibrational relaxation was observed to be significantly slower, with $T_{1}=350 \pm 30$ fs.

A challenge in studying the structural dynamics of proton solvation structures in pure water is that the $\mathrm{OH}$-stretch vibrational lifetimes of water molecules and hydrated protons are extremely short $\left(\sim 0.27 \mathrm{ps}^{20,21}\right.$ and $<100 \mathrm{fs},{ }^{9,22}$ respectively). As a result, the time window in which the structural dynamics can be studied in the vibrationally excited state is very much limited. However, previous studies also showed the presence of slower restructuring and energy dissipation processes following the vibrational relaxation. This observation suggests that the structural lifetime of the proton hydration structures is on the order of picoseconds. ${ }^{23}$

Here, we use femtosecond mid-infrared pump-probe spectroscopy to study the structure and dynamics of proton solvation structures in water/dimethyl sulfoxide (DMSO) mixtures. Dimethyl sulfoxide (DMSO) is a polar, aprotic solvent that solvates water molecules well, but breaks the

Received: July 19, 2018

Revised: September 13, 2018

Published: October 12, 2018 
hydrogen-bond network. DMSO has a quite strong interaction with water and protons, and for a water fraction below $20 \%$, water molecules primarily exist as monomers solvated by DMSO molecules. ${ }^{24}$ This isolation of water molecules can increase the vibrational lifetime up to 5 times compared to bulk water, as has been recently demonstrated in a timeresolved spectroscopy study of the OD-stretch vibrations of $\mathrm{HDO} / \mathrm{H}_{2} \mathrm{O} / \mathrm{DMSO}$ solutions. ${ }^{25}$ In view of this result, studying the dynamics of protons in a mixed water/DMSO solvent can provide important information on the vibrational energy relaxation and structural dynamics of the solvated proton. We compare the results with recent studies on the structural dynamics of solvated protons in water/acetonitrile studies. ${ }^{17,18}$

\section{EXPERIMENTAL SECTION}

Linear mid-infrared absorption spectra were obtained in transmission mode using a commercial Fourier transform spectrometer (Bruker Vertex 80v). The two-color mid-IR pump-probe experiments were carried out using independently tunable femtosecond mid-infrared pump and probe pulses. Pulses were derived from optical parametric amplifiers (OPA), followed by difference-frequency processes. First, a regenerative Ti:sapphire amplifier (Coherent) produces 800 $\mathrm{nm} 35 \mathrm{fs}$ pulses with a pulse energy of $3.3 \mathrm{~mJ}$ at a repetition rate of $1 \mathrm{kHz}$. And $30 \%$ of the $800 \mathrm{~nm}$ light was used to pump a homebuilt OPA, seeded by the white light that is generated by focusing a small fraction of the $800 \mathrm{~nm}$ pulse in a sapphire crystal. A spectral fraction of this white light was amplified by the $800 \mathrm{~nm}$ beam in a three-step OPA process using $\beta$-barium borate $(\mathrm{BBO})$ crystals. This OPA process yielded signal and idler pulses with a total energy of $220 \mu \mathrm{J}$. The signal and idler pulses were mixed in a silver gallium disulfide (AGS) crystal to generate mid-infrared pulses at their difference frequency. These pulses are used as probe pulses. The remainder of the signal and idler was filtered out by a germanium filter. This filter introduces group velocity dispersion (GVD) on the produced infrared pulses. This GVD was compensated by $\mathrm{CaF}_{2}$ windows. We produced tunable $2400-3400 \mathrm{~cm}^{-1}$ pulses with a pulse duration of $\sim 60 \mathrm{fs}$, a spectral full width at half-maximum (fwhm) of $250 \mathrm{~cm}^{-1}$, and an energy between 2 and $5 \mu \mathrm{J}$ depending on frequency. The beam was sent on a $1 \mathrm{~mm}$ wedged $\mathrm{ZnSe}$ window to produce probe $(8 \%)$ and reference beams. The probe beam was sent through a delay stage.

We generated mid-infrared pump pulses with a central frequency of $2550 \mathrm{~cm}^{-1}$ by pumping a commercial OPA (Spectra-Physics) with the remaining $70 \%$ of $800 \mathrm{~nm}$ beam. This OPA employs the same white-light-seeded three-step BBO-based amplification process that we used to generate the probe pulses. The produced signal and idler with a total energy of $\sim 700 \mu \mathrm{J}$ were difference-frequency mixed in a AGS crystal to produce $16 \mu \mathrm{J}$ pulses with a spectral fwhm of $150 \mathrm{~cm}^{-1}$. To generate pump pulses with a central frequency of $2900 \mathrm{~cm}^{-1}$ and higher, the OPA was modified in the following way. After two-step BBO-based amplification, we frequency-doubled the idler in another BBO crystal. The resulting second harmonic of the idler was then used to seed an OPA process in a potassium titanyl phosphate crystal pumped by the rest of the $800 \mathrm{~nm}$ beam. After filtering out all of the visible and near-infrared light with a silicon filter, this process yielded $20 \mu \mathrm{J}$ pulses with a spectral fwhm of $150 \mathrm{~cm}^{-1}$. The polarization of the pump pulse was set at $45^{\circ}$ with respect to that of the probe using a halfwave plate.
The pump and the delayed probe beams were focused in spatial overlap in the sample. The reference beam is used for frequency-resolved pulse-to-pulse normalization of the probe intensity. This beam was also transmitted through the sample, but not in spatial overlap with the pump. After the sample, the probe and reference beams were recollimated, transmitted through a wire-grid polarizer set to select either the parallel or the perpendicular polarization component with respect to the pump polarization, sent to a spectrometer, and detected with a $3 \times 32$ mercury-cadmium-telluride array. By mechanical chopping of each second pump pulse, we measured transient absorption change spectra with probe delays up to $50 \mathrm{ps}$. The time resolution as defined by the cross-correlate of pump and probe was $\sim 0.2$ ps.

From the transient absorption changes of the parallel and perpendicularly polarized probe component (with respect to the pump), we construct the so-called isotropic signal

$$
\Delta \alpha_{\text {iso }}(\nu, t)=\frac{1}{3}\left(\Delta \alpha_{\|}(\nu, t)+2 \Delta \alpha_{\perp}(\nu, t)\right)
$$

This signal is independent of the (re)orientation of the transition dipole moments of the excited molecules.

The samples were prepared by dissolving trifluoromethanesulfonic acid (TfOH, 98\%, Sigma-Aldrich) in water and deuterated dimethyl sulfoxide (DMSO- $d_{6}, 99.9 \%$, SigmaAldrich) mixtures under nitrogen atmosphere. Trifluromethanesulfonic acid (triflic acid) fully dissociates in water or DMSO, and the triflate anion does not react with the solution components. The sample cell consisted of $2 \mathrm{~mm} \mathrm{CaF}$ windows separated by Teflon $10-200 \mu \mathrm{m}$ thick spacers. The sample thickness was chosen such to have a linear absorption of $0.5-1 \mathrm{OD}$ at the central probe frequency. The maximum transient absorption signals were $5-10 \%$.

\section{RESULTS AND DISCUSSION}

Linear Spectra. In Figure 1, we show linear infrared absorption spectra of triflic acid/water/DMSO- $d_{6}$ solutions at

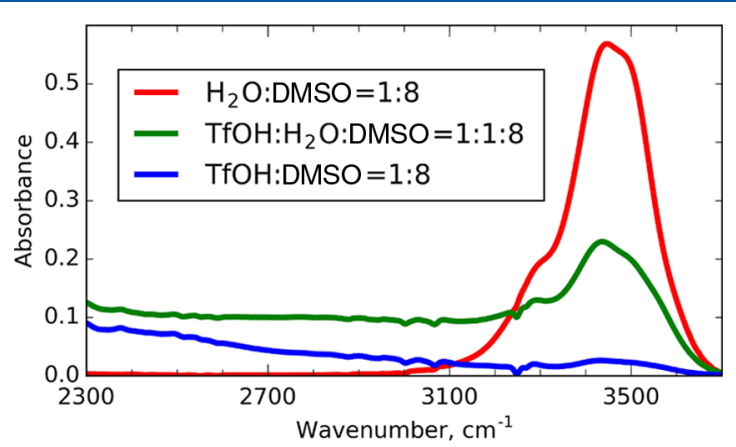

Figure 1. Linear absorption spectra of TfOH/DMSO $=1: 8, \mathrm{TfOH} /$ $\mathrm{H}_{2} \mathrm{O} / \mathrm{DMSO}=1: 1: 8$, and $\mathrm{H}_{2} \mathrm{O} / \mathrm{DMSO}=1: 8$ (the latter is rescaled with respect to the water concentration in the second solution); the DMSO absorption spectrum is subtracted.

different acid/water concentration ratios. All spectra contain a water $\mathrm{OH}$-stretch absorption band at $3200-3700 \mathrm{~cm}^{-1}$. We assign the $3430-3500 \mathrm{~cm}^{-1}$ doublet to the symmetric and antisymmetric water $\mathrm{OH}$-stretch modes of water molecules and the shoulder at $\sim 3300 \mathrm{~cm}^{-1}$ to the overtone of the water bending mode. As the concentrations of water in DMSO are low, water molecules mostly exist as isolated monomers solvated by DMSO. ${ }^{24}$ The samples containing triflic acid have 

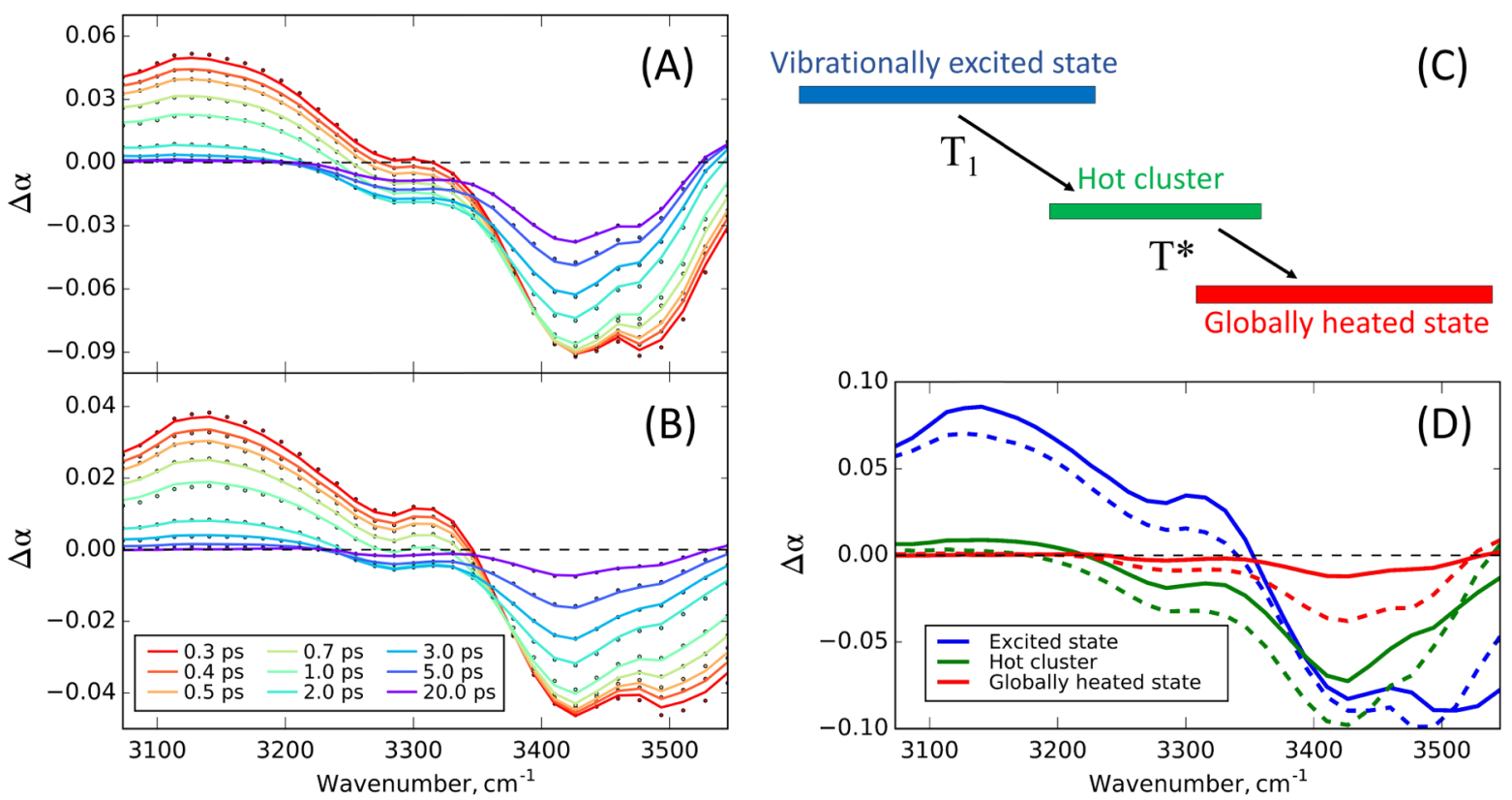

Figure 2. Isotropic pump-probe spectra measured after excitation with a pump pulse centered at $3450 \mathrm{~cm}^{-1}:\left(\right.$ A) $\mathrm{H}_{2} \mathrm{O} / \mathrm{DMSO}=1: 8,(B) \mathrm{TfOH} /$ $\mathrm{H}_{2} \mathrm{O} / \mathrm{DMSO}=1: 1: 8$. The lines represent the result of the fit of the kinetic model to the data. (C) Kinetic model used to fit the isotropic data. (D) The spectral signatures of the different states of the model obtained from the fit: the solid lines represent the solution containing protons (scaled), and the dashed lines represent the solution without protons.

an additional broad absorption continuum at lower frequencies.

In a water/DMSO solution, the proton will be solvated not only by water, but also by DMSO molecules. To understand the contribution of DMSO solvation to the proton absorption continuum, we also recorded the infrared absorption spectrum of triflic acid in dry DMSO. Triflic acid is a superacid and fully dissociates in DMSO solution (confirmed by the absence of $\nu_{\text {as }}\left(\mathrm{SO}_{2}\right)$ band around $\left.1400 \mathrm{~cm}^{-1}\right){ }^{26}$ The long absorption tail at $2300-3300 \mathrm{~cm}^{-1}$ can thus be assigned to the $\mathrm{OH}$-stretch vibrations of protonated DMSO. When the solution contains both water and triflic acid, the absorption continuum has a more flat shape (contrary to the sloping spectrum of (DMSO$\mathrm{H})^{+}$) and a higher intensity. In this case, the continuum absorption will contain the stretch vibrations of (DMSO-H $)^{+}$ and hydrated protons. It has been proposed in the literature that DMSO is more basic than water. ${ }^{27}$ Interestingly, even for solutions containing an excess concentration of protons and only trace amounts of water, the water $\mathrm{OH}$-stretch band with its maximum at $3430 \mathrm{~cm}^{-1}$ is still observed. On the other hand, the intensity of the water $\mathrm{OH}$-stretch band is significantly reduced in acidic DMSO solutions compared to spectra of water/DMSO mixtures without acid. From this observation, we conclude that water and DMSO are similarly basic so that the protons in solution can be solvated by both DMSO and water molecules. The proton will thus be located in between the oxygen atoms of two DMSO molecules, of a DMSO and a water molecule, or of two water molecules.

Vibrational Dynamics of Water Monomers in DMSO. In Figure 2A,B, we present experimental results obtained by exciting water/DMSO solutions with a pulse centered at 3450 $\mathrm{cm}^{-1}$, i.e., in the frequency region of the water $\mathrm{OH}$-stretch absorption.

The transient spectrum of the initially excited state consists of a decreased absorption above $3350 \mathrm{~cm}^{-1}$ and an enhanced absorption at lower frequencies. The decreased absorption is due to the bleaching of the fundamental $\mathrm{v}=0 \rightarrow 1$ transition and the enhanced absorption results from the $\mathrm{v}=1 \rightarrow 2$ excited-state absorption. The transient spectrum clearly shows the signature of the doublet of the symmetric and antisymmetric $\mathrm{OH}$-stretch modes at $3430 / 3500 \mathrm{~cm}^{-1}$ and the $\mathrm{H}_{2} \mathrm{O}$ bending overtone at $3280 \mathrm{~cm}^{-1}$. The excited $\mathrm{v}=1$ state relaxes with time constant $T_{1}=0.84 \pm 0.03$ ps. In previous experiments on the vibrational relaxation of the ODstretch vibration of isotopically dilute $\mathrm{HDO} / \mathrm{H}_{2} \mathrm{O} / \mathrm{DMSO}$ mixtures, two distinctly different relaxation times were observed, corresponding to fast-relaxing red-shifted waterbound HDO molecules and slowly relaxing blue-shifted DMSO-bound HDO molecules. ${ }^{25,28}$ The slow component was assigned to the OD groups of HDO molecules forming hydrogen bonds to the oxygen atom of DMSO, and this component dominated the response for high volume fractions of DMSO. Here, we observe a single vibrational relaxation time for the $\mathrm{OH}$-stretch vibrations of a dilute solution of $\mathrm{H}_{2} \mathrm{O}$ in DMSO. We assign this single component to $\mathrm{H}_{2} \mathrm{O}$ molecules forming hydrogen bonds to the oxygen atoms of DMSO molecules. We thus find that the relaxation time of the $\mathrm{OH}$ stretch vibration of $\mathrm{H}_{2} \mathrm{O}$ in DMSO is $\sim 8$ times faster than that of the OD-stretch vibration of $\mathrm{HDO}$ in DMSO, which is in fact a similar ratio as is observed for neat $\mathrm{H}_{2} \mathrm{O}$ and $\mathrm{HDO} / \mathrm{H}_{2} \mathrm{O}$ ( $\sim 0.27$ vs $1.65 \mathrm{ps})$.

We observe nearly identical transient spectra and relaxation dynamics for solutions with and without added triflic acid, which shows that the excitation pulse excites very little protonated species. We thus conclude that the high-frequency part (above $\sim 3350 \mathrm{~cm}^{-1}$ ) of the linear infrared spectrum represents isolated water molecules, even for solutions that contain equal amounts of water and triflic acid.

The spectral dynamics of $\mathrm{TfOH} / \mathrm{H}_{2} \mathrm{O} / \mathrm{DMSO}=1: 1: 8$ and $\mathrm{H}_{2} \mathrm{O} / \mathrm{DMSO}=1: 8$ solutions are well described by the same kinetic model (Figure 2C). According to this model, the excited state relaxes to an intermediate state that subsequently 
decays to a final thermalized state. This model thus accounts for a delay of the global thermalization with respect to the vibrational relaxation. This model has been used before to describe vibrational relaxation in water and other hydrogenbonded liquids. ${ }^{29,30}$ In Figure 2D, we present the transient spectral components that are obtained from fitting this threestate model to the experimental data.

The transient absorption spectrum of the intermediate state that is reached in the vibrational relaxation shows a bleaching between 3250 and $3550 \mathrm{~cm}^{-1}$. We explain this bleaching from a strong local heating effect. The vibrational relaxation leads to a local dissipation of energy and thus to a weakening of the hydrogen bonds. As a result, the absorption cross section of the $\mathrm{O}-\mathrm{H}$-stretch vibrations decreases. The transient absorption spectrum of the final globally heated state is very similar to that of the intermediate, locally hot cluster, but only the amplitude is much smaller. The intermediate state relaxes with a time constant of $2.6 \pm 0.3 \mathrm{ps}$, irrespective of whether the solution contains protons or not.

The addition of acid does have an effect on the amplitude of the globally heated state spectrum (shown in red). When we scale the spectra with respect to the transient absorption spectrum of the initial excited vibrational state, the amplitude of the final globally heated state has a 2 times higher amplitude for a $\mathrm{H}_{2} \mathrm{O} / \mathrm{DMSO}$ solution than for a $\mathrm{H}^{+} / \mathrm{H}_{2} \mathrm{O} / \mathrm{DMSO}$ solution. This difference can be explained from the fact that in the presence of $\mathrm{H}^{+}$, a large fraction of the $\mathrm{H}_{2} \mathrm{O}$ molecules will become part of a proton solvation structure, thus absorbing at lower frequencies. As a result, the solution contains less isolated $\mathrm{H}_{2} \mathrm{O}$ molecules of which the absorption will be affected by the same heat energy distributed over the solution after the relaxation. As a result, the transient absorption spectrum of the final globally heated state has a smaller amplitude.

Vibrational Dynamics of Protonated Clusters in Water/DMSO Solutions. Transient Spectra. To investigate the structure and dynamics of the solvated proton, we measure transient absorption spectra following excitation of the proton absorption continuum at frequencies $<3200 \mathrm{~cm}^{-1}$. Figure 3 shows isotropic transient spectra of three solutions with different $\mathrm{TfOH} / \mathrm{H}_{2} \mathrm{O} / \mathrm{DMSO}$ ratios obtained with excitation pulses centered at $2550 \mathrm{~cm}^{-1}$ for time delays between pump and probe pulses ranging from 0.26 to 20 ps.

For all three solutions, the transient absorption spectra show quite similar shapes and dynamics. At early delay times, the transient spectrum consists of a broad negative absorption change (bleach) below $3100 \mathrm{~cm}^{-1}$ and a positive absorption change (induced absorption) above $3200 \mathrm{~cm}^{-1}$. In all cases, the negative absorption signal has its maximum at $\sim 2700 \mathrm{~cm}^{-1}$, and the positive absorption signal is centered at $\sim 3400 \mathrm{~cm}^{-1}$. For solutions containing a larger amount of water, we observe a larger negative absorption signal negative shoulder at 3000 $\mathrm{cm}^{-1}$ (Figure 3C).

The spectral shape observed at early delay times (up to $1 \mathrm{ps}$ ) differs from the expected initial shape of a transient absorption spectrum. Usually, the excitation results in a bleach around the frequency of the pump pulse (due to bleaching of the fundamental $\mathrm{v}=0 \rightarrow 1$ transition) and an induced absorption at lower frequency due to $\mathrm{v}=1 \rightarrow 2$ absorption starting from the excited $\mathrm{v}=1$ vibrational state. Here, we observe the induced absorption at higher frequencies than the bleach. Potentially, this could be the result of a positive anharmonicity (frequency of $\mathrm{v}=1 \rightarrow 2$ higher than that of $\mathrm{v}=0 \rightarrow 1$ ), but

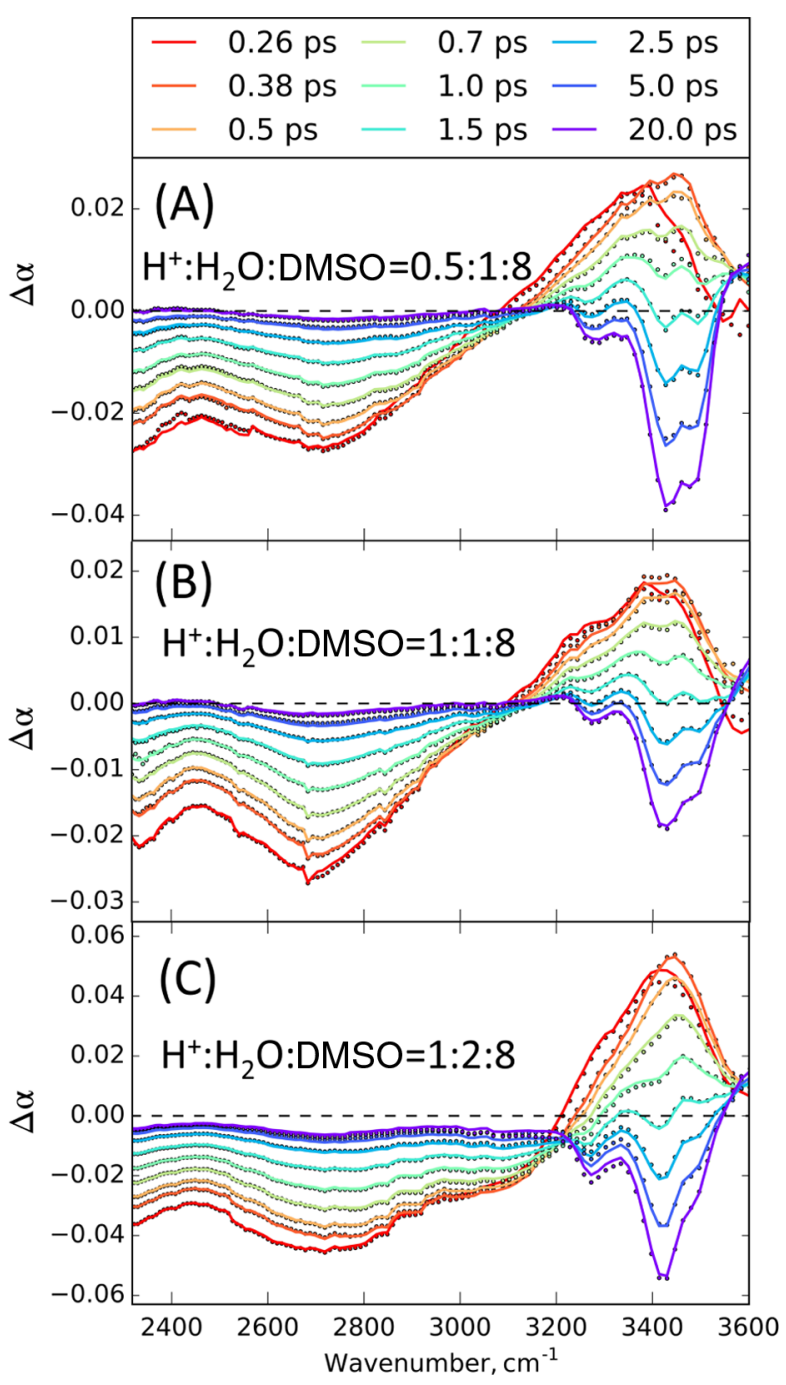

Figure 3. Isotropic pump-probe spectra of triflic acid/water/DMSO$d_{6}$ solutions with respective concentration ratios: (A) $0.5: 1: 8$, (B) $1: 1: 8$, and (C) 1:2:8. Pump pulse is centered at $2550 \mathrm{~cm}^{-1}$. The lines represent the result of the fit.

this is not expected for the $\mathrm{OH}$-stretch vibration of water and protonated water molecules. Moreover, the dynamics of the induced absorption are too slow to be caused by vibrational relaxation of the $\mathrm{v}=1$ state of a strongly hydrogen-bonded $\mathrm{OH}$ group. A positive transient absorption signal at higher frequencies is commonly observed after vibrational relaxation of hydrogen-bonded $\mathrm{OH}$-stretch vibrations. In the vibrational relaxation process, the excitation energy of the $\mathrm{OH}$-stretch vibration is transferred to the hydrogen-bond network. ${ }^{21,29}$ As a result, the hydrogen bonds become longer and weaker, which leads to a blue shift of the $\mathrm{OH}$-stretch absorption spectrum. This blue shift results in a transient spectrum with a bleach in the center and the red wing of the $\mathrm{OH}$-stretch absorption band, and an induced absorption in the blue wing of the $\mathrm{OH}$ stretch absorption band.

We thus conclude that the excited $\mathrm{O}-\mathrm{H}$ vibration rapidly relax on a time scale of $\sim 100$ fs and that the transient spectra are dominated by the local heating effects resulting from this relaxation. The shape of the broad bleaching signal below 3100 $\mathrm{cm}^{-1}$ changes with increasing delay time. This result indicates that the fast vibrational relaxation process not only leads to a local hot state, but that there may be another contributing state 
with a somewhat different associated transient spectrum and different dynamics.

In Figure 4A, we show the absorption change as a function of delay time for three different probing frequencies. The
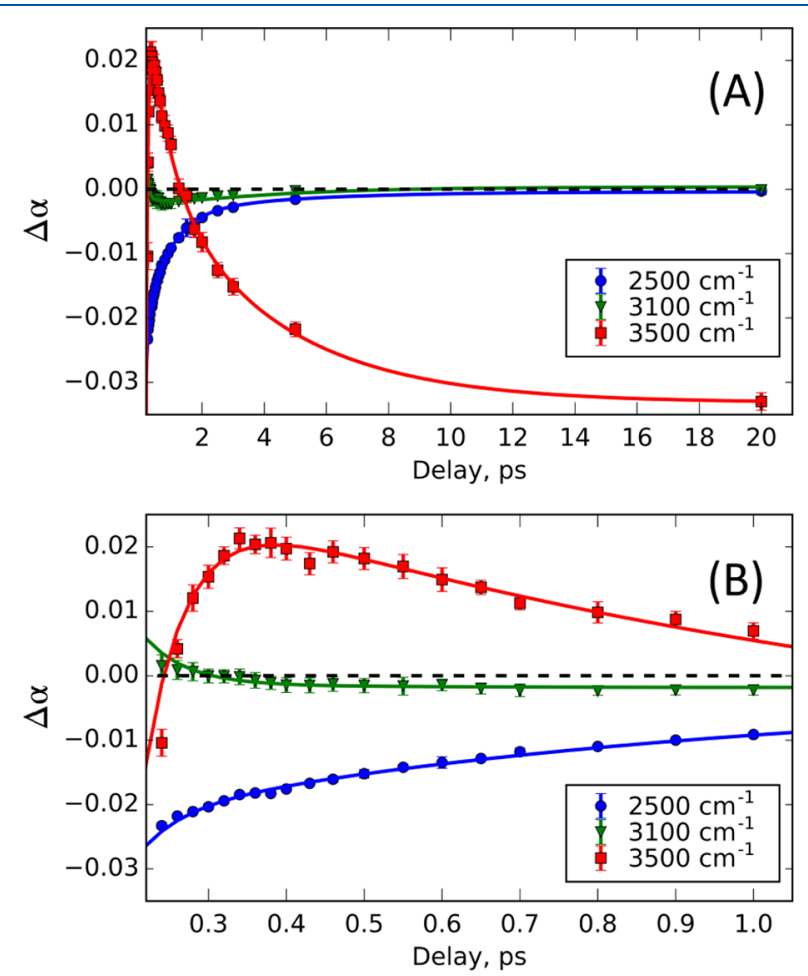

Figure 4. (A) Isotropic pump-probe signal of the solution $\mathrm{TfOH} /$ $\mathrm{H}_{2} \mathrm{O} / \mathrm{DMSO}=0.5: 1: 8$ at different frequencies as a function of time delay. (B) Zoom-in view of the delay time traces of (A) for delay times below 1 ps. The lines are the result of a fit to the kinetic model shown in Figure 5.

transient absorption evolves on a time scale of a few picoseconds to a final bleaching signal (negative absorption change) for frequencies above $3200 \mathrm{~cm}^{-1}$ and a near-zero absorption change at lower frequencies. The signals no longer change after $20 \mathrm{ps,}$ which indicates that these signals correspond to the fully thermalized state. In Figure 4B, we show a zoom-in view of the early delay time range. It is seen that the signal at $3500 \mathrm{~cm}^{-1}$ starts as a bleaching signal, but rapidly evolves into an induced absorption signal on a time scale of $\sim 100 \mathrm{fs}$.
We fit the isotropic spectra for all of the solutions to the kinetic model presented in Figure 5A. Using this kinetic model, we obtained the spectral components shown in Figure 5B. The actual fits of the model to experimental data are represented by the solid lines in Figures 3 and 4.

The decomposed spectra have similar shapes for all three solutions keeping all of the characteristic features (Figure S1). The corresponding time constants are $T_{1}=95 \pm 10 \mathrm{fs}, T^{*}=$ $0.71 \pm 0.04 \mathrm{ps}$, and $\tau_{\mathrm{r}}=4.0 \pm 0.4 \mathrm{ps}$. The nature of the states can be identified from the shape of the associated transient spectra.

Vibrationally Excited State of the Protonated Clusters. We assign the first component (shown in blue in Figure 5B) to the vibrationally excited state, i.e., the occupation of the $\mathrm{v}=1$ state of an $\mathrm{OH}$-stretch vibration, where the $\mathrm{H}$ atom carries (part of) the proton charge. This assignment is supported by several observations. In the first place, the transient spectrum of this state has a bleach signature at the excitation frequency, which is the expected signature, following the excitation of the $\mathrm{v}=1$ state of an $\mathrm{OH}$-stretch vibration. Second, the relaxation of this state is ultrafast $(95 \pm 10 \mathrm{fs})$, in agreement with earlier observations that the vibrational relaxation of the stretch vibrations of strongly hydrogen-bonded $\mathrm{OH}$ groups in protonated species occurs on a time scale of $\sim 100 \mathrm{fs}$ or even shorter. ${ }^{9,22}$ Third, we find this component to be anisotropic (see Supporting Information), as is indeed expected for the transient absorption signal following excitation of the $\mathrm{v}=1$ state. In the case of a (local) heating effect, the anisotropy of the associated spectral change is usually close to zero. Finally, we observe a quite similar transient spectrum at early delay times in pump-probe experiments on a triflic acid/DMSO solution (containing only a trace amount of water) with the same pump pulse centered at $2550 \mathrm{~cm}^{-1}$ (Figure S3). In this case, this spectral component decays somewhat slower $\left(T_{1}=\right.$ $190 \pm 20 \mathrm{fs}$ ). As discussed before, in this solution, the proton will be solvated completely by DMSO molecules and the broad absorption band is due to the $\mathrm{OH}$-stretch vibrations of $(\mathrm{DMSO}-\mathrm{H})^{+}$ions. In these ions, the proton attaches to oxygen atoms of DMSO and the positive charge resides in part on the sulfur atoms. ${ }^{31,32}$

The transient spectrum of the vibrationally excited state also shows a strong response at frequencies $>2800 \mathrm{~cm}^{-1}$. We observe an intense bleach at $3350-3600 \mathrm{~cm}^{-1}$ and a broad induced absorption at $2800-3350 \mathrm{~cm}^{-1}$. This response is similar to the transient spectrum that results from the excitation of the $\mathrm{v}=1$ state of water $\mathrm{OH}$-stretch vibrations. However, the excitation pulse at $2550 \mathrm{~cm}^{-1}$ is far out of
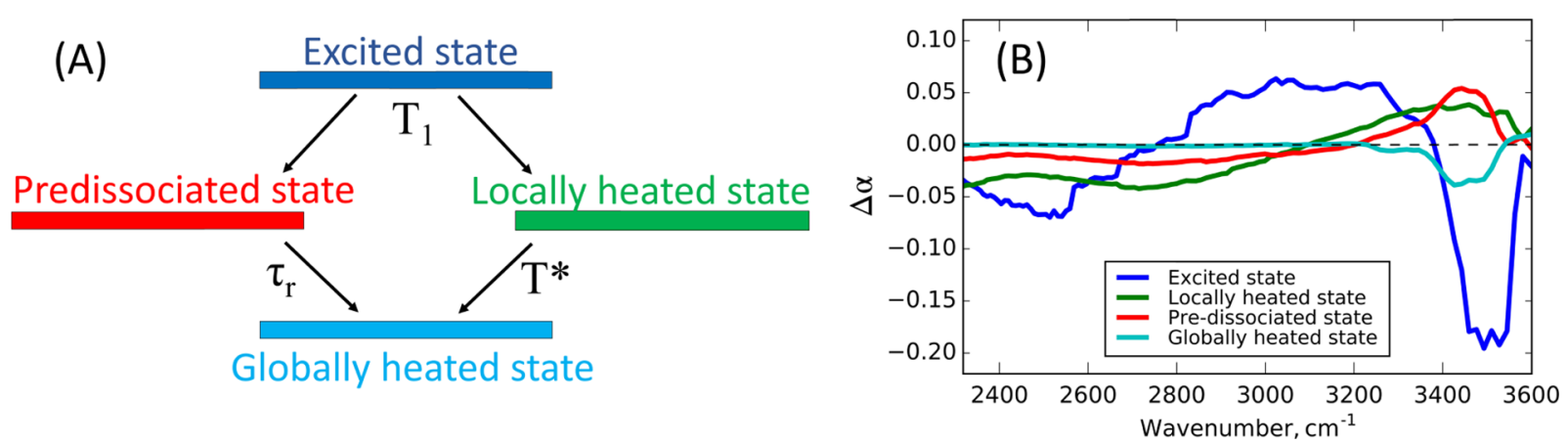

Figure 5. (A) Scheme of the kinetic model, describing the spectral dynamics after excitation at $\nu_{\text {pump }}=2550 \mathrm{~cm}^{-1}$; the arrows illustrate population transfer between the states. (B) The corresponding transient spectra of the states for $[0.5: 1: 8]$ solution. 
resonance with the $\mathrm{OH}$-stretch vibration of isolated nonprotonated water molecules (see $\mathrm{H}_{2} \mathrm{O}$ /DMSO spectrum in Figure 1). Hence, the high-frequency response following excitation of the $\mathrm{OH}$-stretch vibration of a proton solvation structure at $2500 \mathrm{~cm}^{-1}$ likely results from the strong coupling of this mode to the $\mathrm{OH}$-stretch vibrations of the neighboring water molecule. These water molecules can be directly hydrogen-bonded to the proton, e.g., forming a (DMSO$\mathrm{H})^{+}-\mathrm{OH}_{2}$ structure, where the proton is primarily bonded to the oxygen atom of the $\mathrm{S}=\mathrm{O}$ group of the DMSO molecule. Such a structure is reminiscent of an Eigen structure, where $(\mathrm{DMSO}-\mathrm{H})^{+}$plays the role of the central $\mathrm{H}_{3} \mathrm{O}^{+}$. Excitation of the $\mathrm{OH}$ vibration of $(\mathrm{DMSO}-\mathrm{H})^{+}$to the $\mathrm{v}=1$ state can lead to a contraction of the hydrogen bond to the $\mathrm{H}_{2} \mathrm{O}$ molecule. Such a contraction has been observed before for hydrated protons in aqueous Nafion ${ }^{19,33}$ and acetonitrile ${ }^{17}$ and has also been predicted to occur in theoretical studies of infrared-induced proton transfer. ${ }^{6,34}$ A contraction of the (DMSO-H $)^{+}-\mathrm{H}_{2} \mathrm{O}$ hydrogen bond implies that the DMSO- $\mathrm{H}^{+}-\mathrm{H}_{2} \mathrm{O}$ structure becomes more Zundel-like with the proton more equally shared between the flanking DMSO and $\mathrm{H}_{2} \mathrm{O}$ molecules. Such an excitation-induced evolution from Eigen-like to Zundel-like will be accompanied by a red shift of the absorption of the $\mathrm{OH}$-stretch vibrations of the hydrogen-bonded $\mathrm{H}_{2} \mathrm{O}$ molecule. The observed induced absorption signal at $2800-3300 \mathrm{~cm}^{-1}$ agrees with the reported frequency range of the $\mathrm{OH}$-stretch vibrations of $\mathrm{H}_{2} \mathrm{O}$ molecules that flank the Zundel proton in water. $^{7,8,12}$

Local Heating and Vibrational Predissociation. The two states of Figure 5B indicated in red and green are produced by the relaxation of the $\mathrm{v}=1$ state of the proton vibration. The corresponding spectral shapes reflect the effect of a local dissipation of energy. The green spectrum with a decay time constant of $0.71 \pm 0.04$ ps consists of a broad negative absorption (bleaching) at frequencies below $3100 \mathrm{~cm}^{-1}$ and a positive absorption signal at higher frequencies. We assign this smooth featureless spectrum to the result of a local heating process: as a result of the vibrational relaxation, energy is transferred to the hydrogen bonds of the protonated cluster. The weakening of these hydrogen bonds shifts the absorption spectrum of the $\mathrm{OH}$-stretch vibrations to higher frequencies. This local heating effect decays when the local hydrogen bonds transfer their energy to the solvent surrounding the cluster, leading to an equilibration of the heat with the surrounding DMSO matrix. The spectrum of the locally heated cluster depends on the water concentration. For the [1:2:8] solution, we observe a clear rise of a negative shoulder at $3000 \mathrm{~cm}^{-1}$ and a larger intensity of the high-frequency-induced absorption. We explain this shoulder from the larger fraction of water dimers and trimers present in solution. At this higher water concentration, a significant fraction of the protonated DMSO- $\mathrm{H}^{+}-\mathrm{H}_{2} \mathrm{O}$ clusters is hydrogen-bonded to an additional water molecule. In this case, the local heating of the cluster affects two closely spaced, hydrogen-bonded water molecules, of which the $\mathrm{O}-\mathrm{H}$ vibrations absorb at lower frequencies than $\mathrm{H}_{2} \mathrm{O}$ monomers in DMSO. ${ }^{25,28}$ The blue shift of the absorption spectrum of this dimer leads to an additional negative absorption change at $\sim 3100 \mathrm{~cm}^{-1}$ and a positive absorption change around $3400 \mathrm{~cm}^{-1}$.

The spectrum of the other intermediate state indicated in red also consists of a negative absorption part below 3200 $\mathrm{cm}^{-1}$ that is less structured than the bleaching of the locally heated state and an induced absorption at high frequencies.
The shape of this induced absorption is strikingly similar to the linear infrared spectrum of water monomers in DMSO. This induced absorption shows the double-peak structure of water $\mathrm{OH}$-stretch symmetric and antisymmetric modes and a shoulder at $3300 \mathrm{~cm}^{-1}$ due to the water bending overtone. We conclude that the induced absorption corresponds to the creation of additional water monomers (solvated only by DMSO molecules), resulting from the dissociation of the originally excited (DMSO-H $)^{+}-\mathrm{H}_{2} \mathrm{O}$ cluster. Hence, the energy released by the vibrational relaxation is not only redistributed among several low-frequency modes (hydrogen bonds) of the excited cluster, but in some cases, this energy is transferred to a single hydrogen bond between (DMSO-H) ${ }^{+}$ and $\mathrm{H}_{2} \mathrm{O}$, leading to dissociation of this bond and ejection of a water molecule. The intermediate state reached in this relaxation is thus highly nonthermal, as most of the transferred energy resides in one particular hydrogen bond, leading to vibrational predissociation. In a subsequent relaxation process with time constant $\tau_{\mathrm{r}}=4.0 \pm 0.4 \mathrm{ps}$, the energy gets transferred to other local modes, with the result that the (DMSO-H $)^{+}-$ $\mathrm{H}_{2} \mathrm{O}$ cluster recombines and the energy distribution becomes thermal.

To corroborate this interpretation, we compared the transient spectrum of the predissociated state (in red) with the difference spectrum of the sum of the $\mathrm{H}_{2} \mathrm{O} / \mathrm{DMSO}$ and $\mathrm{TfOH} / \mathrm{DMSO}$ spectra on the one hand and the $\mathrm{TfOH} / \mathrm{H}_{2} \mathrm{O}$ / DMSO spectrum on the other hand (in blue). The $\mathrm{H}_{2} \mathrm{O}$ / DMSO and TfOH/DMSO solutions contain $\mathrm{H}_{2} \mathrm{O}$ monomers dissolved in DMSO and (DMSO-H $)^{+}$structures, but not $(\mathrm{DMSO}-\mathrm{H})^{+}-\mathrm{H}_{2} \mathrm{O}$ clusters. The $\mathrm{TfOH} / \mathrm{H}_{2} \mathrm{O} / \mathrm{DMSO}$ solution will contain all of these three components. If the contributions of the spectra are chosen such that the $\mathrm{H}_{2} \mathrm{O}$ / DMSO and $\mathrm{TfOH} / \mathrm{DMSO}$ solutions together represent an equal amount of $\mathrm{H}_{2} \mathrm{O}$ and protons as the $\mathrm{TfOH} / \mathrm{H}_{2} \mathrm{O} / \mathrm{DMSO}$ solution, the difference spectrum $S\left(\mathrm{H}_{2} \mathrm{O} / \mathrm{DMSO}\right)+S(\mathrm{TfOH} /$ DMSO $)-S\left(\mathrm{TfOH} / \mathrm{H}_{2} \mathrm{O} / \mathrm{DMSO}\right)$ will represent the transfer of $(\mathrm{DMSO}-\mathrm{H})^{+}-\mathrm{H}_{2} \mathrm{O}$ clusters into water monomers and $(\mathrm{DMSO}-\mathrm{H})^{+}$structures. In Figure 6, this difference spectrum is compared to the red transient spectrum. It is seen that these spectra are highly similar in shape, thus supporting the interpretation of the red transient spectrum as the result of dissociation of the hydrogen bond between (DMSO-H $)^{+}$and $\mathrm{H}_{2} \mathrm{O}$ clusters of $(\mathrm{DMSO}-\mathrm{H})^{+}-\mathrm{H}_{2} \mathrm{O}$ clusters.

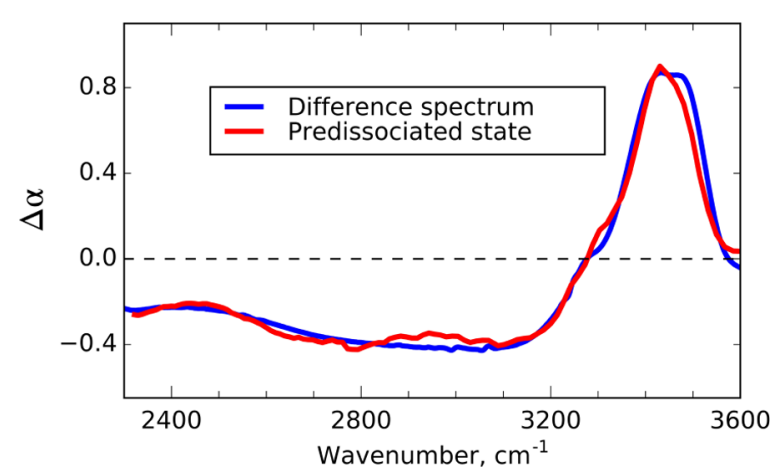

Figure 6. Comparison of the red transient spectrum of Figure $5 \mathrm{~B}$ for $\mathrm{TfOH} / \mathrm{H}_{2} \mathrm{O} / \mathrm{DMSO}=1: 2: 8$ solution with the difference spectrum (blue) $S\left(\mathrm{H}_{2} \mathrm{O} / \mathrm{DMSO}\right)+S(\mathrm{TfOH} / \mathrm{DMSO})-S\left(\mathrm{TfOH} / \mathrm{H}_{2} \mathrm{O} /\right.$ DMSO), where $S$ indicates the linear absorption spectrum of the solution in parentheses. 
The good agreement of the transient spectrum of (DMSO$\mathrm{H})^{+}-\mathrm{H}_{2} \mathrm{O}$ predissociation with the corresponding linear difference spectrum implies that among the possible protonated species, only $(\mathrm{DMSO}-\mathrm{H})^{+}$and $(\mathrm{DMSO}-\mathrm{H})^{+}-$ $\mathrm{H}_{2} \mathrm{O}$ have significant concentrations in the studied solutions. This notion is confirmed by the fact that we can fit the linear infrared spectra of $\mathrm{TfOH} / \mathrm{H}_{2} \mathrm{O} / \mathrm{DMSO}$ solutions of different relative concentrations considering only $\mathrm{H}_{2} \mathrm{O},(\mathrm{DMSO}-\mathrm{H})^{+}$, and (DMSO-H $)^{+}-\mathrm{H}_{2} \mathrm{O}$ as the species present in solution (Figure S5).

The final state (shown in cyan) is the result of the equilibration of the energy over the pump focus. This globally heated state has a transient spectrum that primarily consists of a bleaching signal in the frequency region of the water-inDMSO spectrum. This bleaching reflects the decrease of the cross section of the water $\mathrm{OH}$-stretch vibrations resulting from a weakening of the hydrogen bonds. In the frequency region of the protonated species, the absorption change of the globally heated state has almost zero intensity, which differs from what is observed for the steady-state thermal difference spectrum (Figure S6).

\section{DISCUSSION}

The transient spectrum of the vibrationally excited protonated cluster decays with a time constant of $95 \pm 10$ fs. This decay time is significantly shorter than the vibrational relaxation time of the OH-stretch vibration of $(\mathrm{DMSO}-\mathrm{H})^{+}$in dry DMSO $\left(T_{1}\right.$ $=190 \pm 20 \mathrm{fs}$ ). From this, we conclude that the $\mathrm{H}_{2} \mathrm{O}$ molecule in the (DMSO-H $)^{+}-\mathrm{H}_{2} \mathrm{O}$ cluster provides additional accepting modes for the excited $\mathrm{OH}$-stretch vibration, thus doubling the relaxation rate.

The transient spectrum of the vibrationally excited state of the proton solvated by DMSO and water molecules (blue spectrum of Figure 5B) also shows that the excitation leads to a rapid contraction of the hydrogen bond between (DMSO-H) ${ }^{+}$ and $\mathrm{H}_{2} \mathrm{O}$ within the (DMSO-H $)^{+}-\mathrm{H}_{2} \mathrm{O}$ cluster. Such a rapid contraction of the hydrogen bond within a proton solvation cluster has been observed before for protonated water in the nanochannels of sulfonated perfluoropolymer Nafion. ${ }^{19,33}$ This hydrogen-bond contraction was explained from the fact that the first excited state of the proton vibration possesses a higher energy in the strongly asymmetric potential of the Eigen proton hydration structure than in the more symmetric double-well potential of the Zundel proton hydration structure. Hence, the energy of the $v=1$ state can be lowered by evolving from an Eigen to a Zundel structure, which corresponds to a contraction and strengthening of the hydrogen bond between the $\mathrm{H}_{3} \mathrm{O}^{+}$core and a water molecule in its hydration shell.

In the present case of (DMSO-H $)^{+}-\mathrm{H}_{2} \mathrm{O}$ cluster, we observe a similar vibrational-excitation-induced contraction. Contraction of the hydrogen bond between (DMSO-H) ${ }^{+}$and $\mathrm{H}_{2} \mathrm{O}$ molecule leads to a lowering of the energy of the $\mathrm{v}=1$ state and to a red shift of the absorption spectrum of the $\mathrm{H}_{2} \mathrm{O}$ molecule, thus explaining the observed response in the highfrequency part of the blue transient spectrum of Figure 5B. The transient spectrum resulting from the subsequent vibrational predissociation process (red spectrum of Figure $5 \mathrm{~B}$ ) indicates that the proton vibrational potential of the contracted (DMSO-H $)^{+}-\mathrm{H}_{2} \mathrm{O}$ Zundel-like cluster is not completely symmetric, but still has its lowest well near the oxygen of DMSO. If this potential has been completely symmetric, predissociation of the cluster would likely result in both DMSO and $\mathrm{H}_{3} \mathrm{O}^{+}$fragments and in DMSO- $\mathrm{H}^{+}$and $\mathrm{H}_{2} \mathrm{O}$ fragments. The transient spectrum shows that the predissociation results in the production of $\mathrm{H}_{2} \mathrm{O}$ molecules, which implies that primarily the $\mathrm{H}^{+}-\mathrm{H}_{2} \mathrm{O}$ hydrogen bond dissociates, indicating that this bond is weaker than the $\mathrm{DMSO}-\mathrm{H}^{+}$ hydrogen bond.

The proton solvation in water/DMSO solutions strongly differs from that in water/acetonitrile solutions that have been studied before. ${ }^{13,17,18}$ Acetonitrile is only weakly polar and does not strongly interact with the proton. Hence, in water/ acetonitrile solutions, the protons are primarily solvated by water molecules leading to larger proton-water clusters that are embedded in an acetonitrile matrix. The high level of hydration of protons in water/acetonitrile mixtures is evident from the linear infrared spectrum that shows the presence of water molecules in the second solvation shell. ${ }^{18}$ The larger hydration shell of the proton in acetonitrile solution likely explains the observation that the vibrational relaxation of the $\mathrm{OH}$-stretch vibration of the hydrated proton is significantly faster in acetonitrile $\left(T_{1}<65 \mathrm{fs}\right)$ than in DMSO $\left(T_{1}=95 \pm 10\right.$ fs). The comparison between the relaxation of the $\mathrm{OH}$-stretch vibration of $(\mathrm{DMSO}-\mathrm{H})^{+}-\mathrm{H}_{2} \mathrm{O}$ and $(\mathrm{DMSO}-\mathrm{H})^{+}\left(T_{1}=190 \pm\right.$ $20 \mathrm{fs}$ ) shows that already one additional water molecule leads to a significant speedup of the relaxation. Hence, it is to be expected that the further solvation with more water molecules in water/acetonitrile will increase the vibrational relaxation rate of the $\mathrm{OH}$-stretch vibration of the proton even further.

At longer delay times, the transient spectrum of the solvated proton in water/DMSO is quite similar to that of the hydrated proton in acetonitrile. In both cases, the isotropic transient spectrum after $\sim 0.5$ ps consists of an induced absorption at high frequencies and a broad bleach at lower frequencies. For both systems, this signal is partly due to a local heating effect and partly due to vibrational predissociation of the hydrated proton cluster. ${ }^{17} \mathrm{~A}$ difference is that the induced absorption of the predissociated water monomers in acetonitrile was relatively featureless: the characteristic doublet of the symmetric and antisymmetric $\mathrm{OH}$-stretch modes of water monomers was not observed, in contrast to the present observations for solvated protons in water/DMSO.

\section{CONCLUSIONS}

We investigated the vibrational dynamics of different mixtures of water, trifluoromethanesulfonic acid $(\mathrm{TfOH})$, and excess dimethyl sulfoxide (DMSO) using two-color femtosecond mid-infrared spectroscopy. We studied mixtures with compositions of $\left[\mathrm{H}^{+}\right] /\left[\mathrm{H}_{2} \mathrm{O}\right] /[\mathrm{DMSO}]=0.5: 1: 8,1: 1: 8$, and $1: 2: 8$. The TfOH acid completely dissociates, leading to the formation of (DMSO-H $)^{+}$and (DMSO-H $)^{+}-\mathrm{H}_{2} \mathrm{O}$ clusters. We find that for all studied mixtures, the solution contains a significant fraction of water monomers, i.e., $\mathrm{H}_{2} \mathrm{O}$ molecules that are solvated by DMSO molecules only and that are not interacting with a proton or with other water molecules. The $\mathrm{OH}$-stretch vibration of these water monomers can be selectively excited with an excitation pulse of $3450 \mathrm{~cm}^{-1}$. The vibrational relaxation dynamics is a two-step process that is independent of the presence of protons in the solution. In the first step, the excited $\mathrm{OH}$-stretch vibration relaxes with a time constant of $0.84 \pm 0.03$ ps to an intermediate state. This intermediate state represents a strong local heating effect, corresponding to the excitation of low-frequency modes (hydrogen bonds) close to the excited $\mathrm{OH}$-stretch vibration. The intermediate state relaxes with a time constant of $2.6 \pm 0.3$ 
ps, leading to a small global heating effect of the sample in the focus of the excitation pulse.

We studied the vibrational dynamics of the protonated structures by exciting the solutions with an excitation pulse centered at $2550 \mathrm{~cm}^{-1}$. Kinetic modeling of the transient spectra shows that this excitation results in a short-living transient absorption spectrum associated with the $\mathrm{v}=1$ state of $(\mathrm{DMSO}-\mathrm{H})^{+}$and $(\mathrm{DMSO}-\mathrm{H})^{+}-\mathrm{H}_{2} \mathrm{O}$ clusters. This transient spectrum consists of a bleaching of the fundamental transition $\mathrm{v}=0 \rightarrow 1$ transition at frequencies $<2800 \mathrm{~cm}^{-1}$, an induced absorption at $2800-3300 \mathrm{~cm}^{-1}$, and a bleaching signal at frequencies $>3300 \mathrm{~cm}^{-1}$. We assign the latter two signals to a strong red shift of the $\mathrm{OH}$-stretch vibrations of water molecules that are close to the excited $\mathrm{O}-\mathrm{H}$ vibration, e.g., in $(\mathrm{DMSO}-\mathrm{H})^{+}-\mathrm{H}_{2} \mathrm{O}$ clusters. The excitation to the $\mathrm{v}=1$ state leads to a contraction of the hydrogen bond between (DMSO-H) ${ }^{+}$and $\mathrm{H}_{2} \mathrm{O}$, thus making the (DMSO-H) ${ }^{+}-\mathrm{H}_{2} \mathrm{O}$ cluster more Zundel-like. Due to this contraction, the frequencies of the $\mathrm{OH}$-stretch vibrations of the $\mathrm{H}_{2} \mathrm{O}$ molecules flanking the proton shift from 3400-3500 to 2800-3300 $\mathrm{cm}^{-1}$.

The excited $\mathrm{v}=1$ state relaxes with $T_{1}=95 \pm 10$ fs. This relaxation leads to two distinctly different intermediate states. One of these states is a locally heated state which is of similar nature to the intermediate state that is created by excitation and relaxation of water monomers in DMSO. The locally hot state relaxes with a time constant of $0.71 \pm 0.04$ ps. The second intermediate state is a vibrational predissociation state. The vibrational energy of the excited $\mathrm{OH}$-stretch vibration of the solvated proton can also be primarily transferred to the hydrogen bond between (DMSO-H) ${ }^{+}$and $\mathrm{H}_{2} \mathrm{O}$ of a (DMSO$\mathrm{H})^{+}-\mathrm{H}_{2} \mathrm{O}$ cluster, leading to dissociation of this bond. This dissociation leads to the transient creation of water monomers. The predissociation state relaxes with a time constant of $4.0 \pm$ $0.4 \mathrm{ps}$, which implies that the hydrogen bond between (DMSO-H $)^{+}$and $\mathrm{H}_{2} \mathrm{O}$ reforms with this time constant.

\section{ASSOCIATED CONTENT}

\section{S Supporting Information}

The Supporting Information is available free of charge on the ACS Publications website at DOI: 10.1021/acs.jpcb.8b06938.

Isotropic transient spectral components for the solutions $\mathrm{TfOH} / \mathrm{H}_{2} \mathrm{O} / \mathrm{DMSO}=1: 1: 8$ and $1: 2: 8$; discussion of the anisotropy time dependence; transient spectra of the dry $\mathrm{TfOH} / \mathrm{DMSO}=1: 8$ solution; comparison of the predissociated state transient spectra for the solutions $\mathrm{TfOH} / \mathrm{H}_{2} \mathrm{O} / \mathrm{DMSO}=1: 1: 8$ and $1: 2: 8$ with the corresponding difference spectra $S\left(\mathrm{H}_{2} \mathrm{O} / \mathrm{DMSO}\right)+$ $S(\mathrm{TfOH} / \mathrm{DMSO})-S\left(\mathrm{TfOH} / \mathrm{H}_{2} \mathrm{O} / \mathrm{DMSO}\right)$; spectral decomposition of the linear infrared spectra of different $\mathrm{TfOH} / \mathrm{H}_{2} \mathrm{O} / \mathrm{DMSO}$ solutions; comparison of the globally heated state transient spectra and the linear thermal difference spectra; and transient spectral dynamics following the excitation at $3200 \mathrm{~cm}^{-1}$ (PDF)

\section{AUTHOR INFORMATION}

\section{Corresponding Author}

*E-mail: sofronov@amolf.nl. Phone: +31207547100.

\section{ORCID}

Oleksandr O. Sofronov: 0000-0001-7744-6404

Huib J. Bakker: 0000-0003-1564-5314

\section{Notes}

The authors declare no competing financial interest.

\section{ACKNOWLEDGMENTS}

This work is part of the research program of the Netherlands Organization for Scientific Research (NWO) and was performed at the research institute AMOLF. This project received funding from the European Research Council (ERC) under the European Union's Horizon 2020 research and innovation program (grant agreement no. 694386). The authors thank Dr. Jan Versluis for the experimental support.

\section{REFERENCES}

(1) Reed, C. A. Myths about the Proton. The Nature of $\mathrm{H}+$ in Condensed Media. Acc. Chem. Res. 2013, 46, 2567-2575.

(2) Stoyanov, E. S.; Stoyanova, I. V.; Reed, C. A. The Unique Nature of $\mathrm{H}+$ in Water. Chem. Sci. 2011, 2, 462-472.

(3) Agmon, N. The Grotthuss Mechanism. Chem. Phys. Lett. 1995, 244, 456-462.

(4) Cukierman, S. Et Tu, Grotthuss! And Other Unfinished Stories. Biochim. Biophys. Acta, Bioenerg. 2006, 1757, 876-885.

(5) Knight, C.; Voth, G. A. The Curious Case of the Hydrated Proton. Acc. Chem. Res. 2012, 45, 101-109.

(6) Ando, K.; Hynes, J. T. Molecular Mechanism of HF Acid Ionization in Water: An Electronic Structure-Monte Carlo Study. J. Phys. Chem. A 1999, 103, 10398-10408.

(7) Kim, J.; Schmitt, U. W.; Gruetzmacher, J. A.; Voth, G. A.; Scherer, N. E. The Vibrational Spectrum of the Hydrated Proton: Comparison of Experiment, Simulation, and Normal Mode Analysis. J. Chem. Phys. 2002, 116, 737-746.

(8) Xu, J.; Zhang, Y.; Voth, G. A. Infrared Spectrum of the Hydrated Proton in Water. J. Phys. Chem. Lett. 2011, 2, 81-86.

(9) Thämer, M.; De Luigi, M.; Ramasesha, K.; Mandal, A.; Tokmakoff, A. Ultrafast 2D IR Spectroscopy of the Excess Proton in Liquid Water. Science 2015, 350, 78-82.

(10) Biswas, R.; Carpenter, W.; Fournier, J. A.; Voth, G. A.; Tokmakoff, A. IR Spectral Assignments for the Hydrated Excess Proton in Liquid Water. J. Chem. Phys. 2017, 146, No. 154507.

(11) Wolke, C. T.; Fournier, J. A.; Dzugan, L. C.; Fagiani, M. R.; Odbadrakh, T. T.; Knorke, H.; Jordan, K. D.; McCoy, A. B.; Asmis, K. R.; Johnson, M. A. Spectroscopic Snapshots of the Proton-Transfer Mechanism in Water. Science 2016, 354, 1131-1135.

(12) Headrick, J. M.; Diken, E. G.; Walters, R. S.; Hammer, N. I.; Christie, R. A.; Cui, J.; Myshakin, E. M.; Duncan, M. A.; Johnson, M. A.; Jordan, K. D. Chemistry: Spectral Signatures of Hydrated Proton Vibrations in Water Clusters. Science 2005, 308, 1765-1769.

(13) Dahms, F.; Fingerhut, B. P.; Nibbering, E. T. J.; Pines, E.; Elsaesser, T. Large-Amplitude Transfer Motion of Hydrated Excess Protons Mapped by Ultrafast 2D IR Spectroscopy. Science 2017, 357, 491-495.

(14) Daly, C. A., Jr.; Streacker, L. M.; Sun, Y.; Pattenaude, S. R.; Hassanali, A. A.; Petersen, P. B.; Corcelli, S. A.; Ben-Amotz, D. Decomposition of the Experimental Raman and Infrared Spectra of Acidic Water into Proton, Special Pair, and Counterion Contributions. J. Phys. Chem. Lett. 2017, 8, 5246-5252.

(15) Kulig, W.; Agmon, N. A "clusters-in-Liquid" Method for Calculating Infrared Spectra Identifies the Proton-Transfer Mode in Acidic Aqueous Solutions. Nat. Chem. 2013, 5, 29-35.

(16) Agmon, N.; Bakker, H. J.; Campen, R. K.; Henchman, R. H.; Pohl, P.; Roke, S.; Thämer, M.; Hassanali, A. Protons and Hydroxide Ions in Aqueous Systems. Chem. Rev. 2016, 116, 7642-7672.

(17) Ottosson, N.; Liu, L.; Bakker, H. J. Vibrational Relaxation of the Aqueous Proton in Acetonitrile: Ultrafast Cluster Cooling and Vibrational Predissociation. J. Phys. Chem. B 2016, 120, 7154-7163.

(18) Dahms, F.; Costard, R.; Pines, E.; Fingerhut, B. P.; Nibbering, E. T. J.; Elsaesser, T. The Hydrated Excess Proton in the Zundel Cation H5O2+: The Role of Ultrafast Solvent Fluctuations. Angew. Chem., Int. Ed. 2016, 55, 10600-10605. 
(19) Liu, L.; Bakker, H. J. Infrared-Activated Proton Transfer in Aqueous Nafion Proton-Exchange- Membrane Nanochannels. Phys. Rev. Lett. 2014, 112, No. 258301.

(20) Van Der Post, S. T.; Hsieh, C. S.; Okuno, M.; Nagata, Y.; Bakker, H. J.; Bonn, M.; Hunger, J. Strong Frequency Dependence of Vibrational Relaxation in Bulk and Surface Water Reveals SubPicosecond Structural Heterogeneity. Nat. Commun. 2015, 6, No. 8384.

(21) Ramasesha, K.; De Marco, L.; Mandal, A.; Tokmakoff, A. Water Vibrations Have Strongly Mixed Intra- and Intermolecular Character. Nat. Chem. 2013, 5, 935-940.

(22) Woutersen, S.; Bakker, H. J. Ultrafast Vibrational and Structural Dynamics of the Proton in Liquid Water. Phys. Rev. Lett. 2006, 96, No. 138305.

(23) Carpenter, W. B.; Fournier, J. A.; Lewis, N. H. C.; Tokmakoff, A. Picosecond Proton Transfer Kinetics in Water Revealed with Ultrafast IR Spectroscopy. J. Phys. Chem. B 2018, 122, 2792-2802.

(24) Wallace, V. M.; Dhumal, N. R.; Zehentbauer, F. M.; Kim, H. J.; Kiefer, J. Revisiting the Aqueous Solutions of Dimethyl Sulfoxide by Spectroscopy in the Mid- and Near-Infrared: Experiments and CarParrinello Simulations. J. Phys. Chem. B 2015, 119, 14780-14789.

(25) Lotze, S.; Groot, C. C. M.; Vennehaug, C.; Bakker, H. J. Femtosecond Mid-Infrared Study of the Dynamics of Water Molecules in Water-Acetone and Water-Dimethyl Sulfoxide Mixtures. J. Phys. Chem. B 2015, 119, 5228-5239.

(26) Leuchs, M.; Zundel, G. Easily Polarizable Hydrogen Bonds in Aqueous Solutions of Acids. Perchloric Acid and Trifluoromethane Sulphonic Acid. J. Chem. Soc., Faraday Trans. 2 1978, 74, 2256-2267.

(27) Rossini, E.; Knapp, E. W. Proton Solvation in Protic and Aprotic Solvents. J. Comput. Chem. 2016, 37, 1082-1091.

(28) Wong, D. B.; Sokolowsky, K. P.; El-Barghouthi, M. I.; Fenn, E. E.; Giammanco, C. H.; Sturlaugson, A. L.; Fayer, M. D. Water Dynamics in Water/DMSO Binary Mixtures. J. Phys. Chem. B 2012, 116, 5479-5490.

(29) Lock, A. J.; Woutersen, S.; Bakker, H. J. Ultrafast Energy Equilibration in Hydrogen-Bonded Liquids. J. Phys. Chem. A 2001, 105, 1238-1243.

(30) Rezus, Y. L. A.; Bakker, H. J. Orientational Dynamics of Isotopically Diluted $\mathrm{H} 2 \mathrm{O}$ and D2O. J. Chem. Phys. 2006, 125, No. 144512.

(31) Tureček, F. Proton Affinity of Dimethyl Sulfoxide and Relative Stabilities of $\mathrm{C} 2 \mathrm{H} 6 \mathrm{OS}$ Molecules and C2H7OS+ Ions. A Comparative G2(MP2) Ab Initio and Density Functional Theory Study. J. Phys. Chem. A 1998, 102, 4703-4713.

(32) Rasul, G.; Prakash, G. K. S.; Olah, G. A. Protonated and Methylated Dimethyl Sulfoxide Cations and Dications. DFT/GIAOMP2 NMR Studies and Comparison with Experimental Data. J. Org. Chem. 2000, 65, 8786-8789.

(33) Liu, L.; Bakker, H. J. Vibrational Excitation Induced Proton Transfer in Hydrated Nafion Membranes. J. Phys. Chem. B 2015, 119, $2628-2637$.

(34) Hammes-Schiffer, S.; Tully, J. C. Vibrationally Enhanced Proton Transfer. J. Phys. Chem. 1995, 99, 5793-5797. 